\title{
Smart Home Appliances through Android (SHATA)
}

\author{
Azman Bin Talib, Norazlina Binti Ahmad, and Ismail Bin Asis
}

\begin{abstract}
This paper presents the overall design of Smart Home Appliances through Android (SHATA) with low cost and wireless remote control. This system is designed to assist and provide support in order to fulfill the needs of elderly and disabled in home and also people who always travel so they can manage the household electrical appliances remotely. Also, the smart home concept in the system improves the standard living at home. The main control system implements wireless Wi-Fi technology to provide remote access from PC/laptop or smart phone to the controller. The design remains the existing electrical switches and provides more safety control on the switches. The switches status is synchronized in all the control system whereby every user interface indicates the real time existing switches status. The system intended to control electrical appliances and devices in house with relatively low cost design, user-friendly interface and ease of installation.
\end{abstract}

Keywords---Home automation, smart home, home appliances, Wi-Fi

\section{INTRODUCTION}

The "Home Automation" concept has existed for many years. The terms "Smart Home", "Intelligent Home" followed and has been used to introduce the concept of networking appliances and devices in the house. Smart Home Appliances through Android (SHATA) represents a great research opportunity in creating new fields in engineering, architecture and computing. This system can support to control electrical appliances switch to turn "ON" and "OFF".

Smart Home becoming popular nowadays and enterquickly in this emerging market. However, these systems are not always accepted by end users, especially due to their complexity and cost. Traditional home automation systems involve the control of electrical appliances which provide the functions such as heating, lighting and shading. But due to the rapid growth of information technology and modern entertainment systems in recent years, these primary functions are expected to be enriched with additional services.

Department of Electrical Engineering, Politeknik Kota Kinabalu, Sabah, Malaysia, azmant@polikk.edu.my

Department of Electrical Engineering, Politeknik Kota Kinabalu, Sabah, Malaysia, norazlina@polikk.edu.my

Department of Electrical Engineering, Politeknik Kota Kinabalu, Sabah, Malaysia, ismail@polikk.edu.my
The benefits of smart home automation systems (the smart house systems) are listed as safety, comfort, power saving and communications. As the systems provide these benefits, some technical requirements must be also respected such as low cost, plug and play, flexibility, easiness of use and reliability. In this study, a smart home automation system, provide an alternative user friendly interface. The system is built to serve multiple users, using up-to-date and emerging technologies, in order to support the communication between the main hardware components of the system. In the following sections, the details of the proposed system architecture are given.

\section{RELATED WORK}

There has been a significant research and numerous approaches for the home automation systems. X10 industry standard, developed in 1975 for communication between electronic devices, is identified as the oldest standard in home automation systems by providing limited control over household devices through the home's power lines. Recently the home automation becomes a popular field of research by the introduction of different technologies such as remote control systems, network enabled devices and internet technologies.

A Bluetooth based home automation system is presented. The system involves a primary controller and a set of Bluetooth sub-controllers in which each controller is physically connected to an individual home device. The sub-controllers are responsible to send all messages to primary controller. Although the system reduces physical wiring through the use of Bluetooth technology it has the disadvantage of incurring an access delay due to the sharing of a single Bluetooth module between numerous devices.

In a Java based home automation system developed, an embedded board which is integrated into a personal computer based web server is physically connected to all home devices. Java technology used in the system provides a built in security. However, the use of a high end computer and the wired installation per home increases the expense of the system.

An interesting system, phone based remote control system, is proposed. Unlike the numerous systems using the internet, the communication in the system is all performed over a fixed telephone line. The advantage of the system is that it can be accessed via any telephone. On the other hand, the lack of graphical user interface, the necessity to remember the user access code and the device codes can be listed as the disadvantages.

After discussing the potential of ZigBee standards in home automation systems, a home automation system based on ZigBee and Wi-Fi network is presented and a virtual home is constructed. It is reported in the study that the ZigBee tech- 
nology has advantages such as lowering the expense of the system and intrusiveness of the respective system installation compared to existing technologies over the virtual home.

In addition to aforementioned systems which offer different technologies in construction of home automation systems, in several other studies, different techniques are presented to improve the home automation. In a data mining method is presented. In the proposed method, regularly occurring device usage patterns are discovered to improve the system performance.

This paper focused more on Wi-Fi technology as the communication medium between the android and the controller which control the electrical appliances.

\section{Methodology}

\section{A. Wi-Fi}

$\mathrm{Wi}-\mathrm{Fi}$, also spelled Wifi or $\mathrm{WiFi}$, is a local area wireless technology that allows an electronic device to exchange data or connect to the internet using $2.4 \mathrm{GHz} \mathrm{UHF}$ and $5 \mathrm{GHz} \mathrm{SHF}$ radio waves. The name is a trademark name (Fig. 1), and is a play on the audiophile term Hi-Fi. The Wi-Fi Alliance defines Wi-Fi as any "wireless local area network (WLAN) products that are based on the Institute of Electrical and Electronics Engineers' (IEEE) 802.11 standards". However, since most modern WLANs are based on these standards, the term "Wi-Fi" is used in general English as a synonym for "WLAN". Only Wi-Fi products that complete Wi-Fi Alliance interoperability certification testing successfully may use the "Wi-Fi CERTIFIED" trademark.

Many devices can use Wi-Fi, e.g., personal computers, video-game consoles, smartphones, some digital cameras, tablet computers and digital audio players. These can connect to a network resource such as the Internet via a wireless network access point. Such an access point (or hotspot) has a range of about 20 meters (66 feet) indoors and a greater range outdoors. Hotspot coverage can comprise an area as small as a single room with walls that block radio waves, or as large as many square kilometres achieved by using multiple overlapping access points.

Depiction of a device sending information wirelessly to another device, both connected to the local network, in order to print a document. Wi-Fi can be less secure than wired connections (such as Ethernet) because an intruder does not need a physical connection. Web pages that use SSL are secure but unencrypted internet access can easily be detected by intruders. Because of this, Wi-Fi has adopted various encryption technologies. The early encryption WEP, proved easy to break. Higher quality protocols (WPA, WPA2) were added later. An optional feature added in 2007, called Wi-Fi Protected Setup (WPS), had a serious flaw that Allowed an attacker to recover the router's password. The Wi-Fi Alliance has since updated its test plan and certification program to ensure all newly certified devices resist attacks.

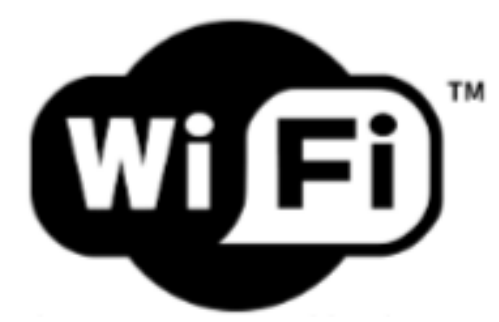

Fig. 1 The Wi-Fi logo used by the Wi-Fi Alliance

\section{B. Android}

Android is a mobile operating system (OS) based on the Linux kernel and currently developed by Google (Fig. 2). With a user interface based on direct manipulation, Android is designed primarily for touchscreen mobile devices such as smartphones and tablet computers, with specialized user interfaces for televisions (Android TV), cars (Android Auto), and wrist watches (Android Wear). The OS uses touch inputs that loosely correspond to real-world actions, like swiping, tapping, pinching, and reverse pinching to manipulate on-screen objects, and a virtual keyboard. Despite being primarily designed for touchscreen input, it also has been used in game consoles, digital cameras, and other electronics.

Android is the most popular mobile OS. As of 2013, Android devices sell more than Windows, iOS, and Mac OS devices combined, with sales in 2012, 2013 and 2014 close to the installed base of all PCs. As of July 2013 the Google Play store has had over 1 million Android apps published, and over 50 billion apps downloaded. A developer survey conducted in April-May 2013 found that $71 \%$ of mobile developers develop for Android. At Google I/O 2014, the company revealed that there were over 1 billion active monthly Android users (that have been active for 30 days), up from 538 million in June 2013.

Android's source code is released by Google under open source licenses, although most Android devices ultimately ship with a combination of open source and proprietary software. Initially developed by Android, Inc., which Google backed financially and later bought in 2005, Android was unveiled in 2007 along with the founding of the Open Handset Alliance a consortium of hardware, software, and telecommunication companies devoted to advancing open standards for mobile devices.

Android is popular with technology companies which require a ready-made, low-cost and customizable operating system for high-tech devices. Android's open nature has encouraged a large community of developers and enthusiasts to use the open-source code as a foundation for community-driven projects, which add new features for advanced users or bring Android to devices which were officially released running other operating systems. The operating system's success has made it a target for patent litigation as part of the so-called "smartphone wars" between technology companies. 


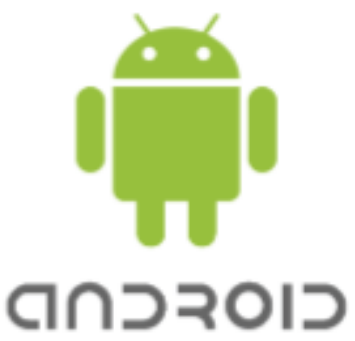

Fig. 2 Android logo

\section{SYSTEM ARCHITECTURE}

The whole infrastructure design as a top-view communication diagram of the home automation system is given in Fig. 3. The system mainly and simply involves the following three components: internet (Wi-Fi), smartphone (android), and a controller.

The controller device function is to connect to the household appliances. The Wi-Fi internet offers service to connect the controller to the android smartphone device. Indeed, the Wi-Fi internet is used for supporting the bidirectional communication between smartphone device and the controller.

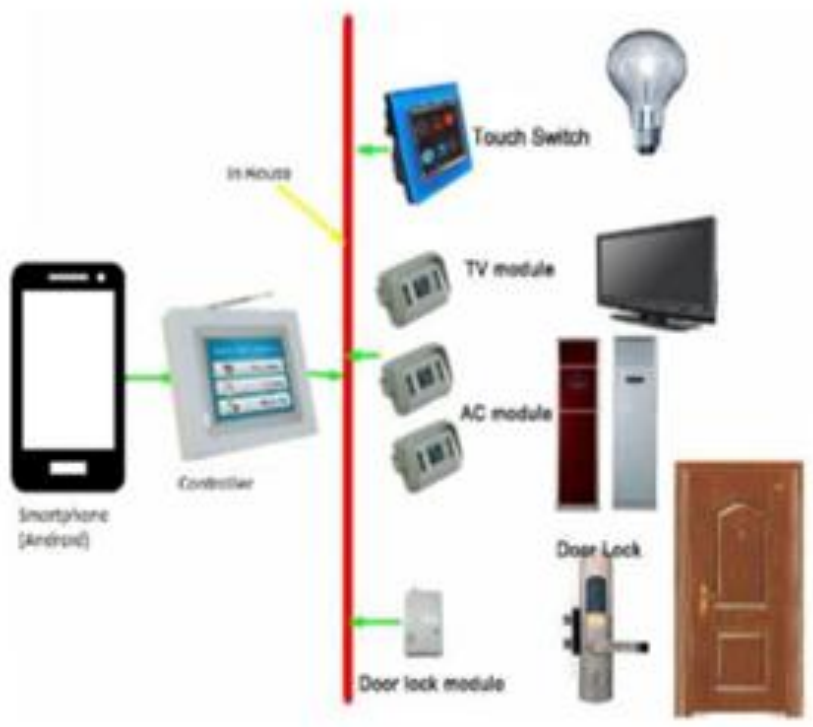

Fig. 3 Communication infrastructure of the proposed system

The mobile smart device running Android operating system, such as smart phone or tablet, on which the Android application software of the system is installed to make the mobile customers contact with and manage the in home devices via the controller. The Android application on the mobile smart device also provides its users with a user friendly and non-complex graphical interface to easily control the automated machines at home. The rest of this section dissects the three main components of the system regarding their tasks and functionalities. The ready to use applications such as "Splashtop" and "Teamviewer" apps can be very useful to smartphone to communicate to the controller (Fig. 4)

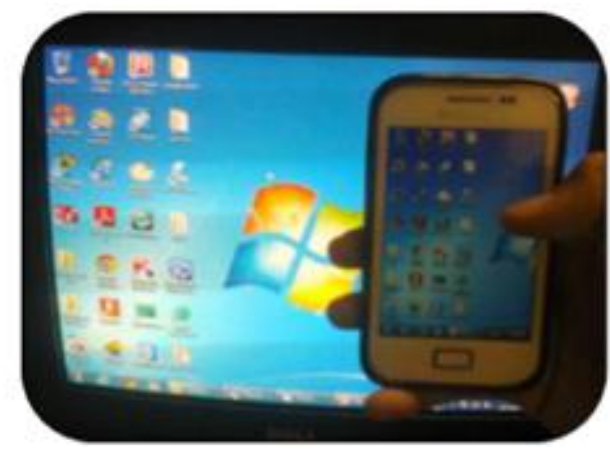

Fig. 4 Android apps interface with controller

\section{SySTEM ANALYSIS}

At the initial phases of the whole project, the main criteria of an appropriate home automation system have been delivered. These criteria may be relative to different designers based on different analysis of the system. All considered properties or attributes for a convenient home automation system are taken into account. The running implementation of the prototype system is also briefly evaluated based on those criteria. After testing the system with different users for sever al times, the evaluation results are considered satisfy. This is due to some technical problems such as low speed in internet connection and some intermittence current at the PCB.

\section{CONCLUSION}

The paper proposes an intelligent automation system using Wi-Fi and Android operating system as the emerging technologies used in home automation area. The SHATA system mainly and simply involves the following three components: internet (Wi-Fi), smartphone (android), and a controller.

The functionalities of each different component of the system are dissected and the communication infrastructures of the parts are explained. According to the evaluation results, the proposed home automation system is adequate in overall.

In conclusion, this low cost system is designed to improve the standard living in home. The remote control function by smart phone provides help and assistance especially to disabled and elderly and also people who always travel so they can manage the household electrical appliances remotely.

\section{REFERENCES}

[1] Bromley K., Perry M., and Webb G.(2003) Connectivity and Services, www.nextwave.org.uk. Trends in Smart Home Systems.

[2] Kovatsch M., Weiss M., and Guinard D. (2010) Proc. of ETFA. Embedding internet technology for home Automation 1-8.

[3] Moraes F., Amory A., Calazans N., Bezerra E., and Petrini J. (2001), 14th Symposium on Integrated Circuits and Systems Design. Using the CAN protocol and reconfigurable computing technology for Web-based smart house automation 
[4] Gill K., Yang S.-H., Yao F., and Lu X. (2009) IEEE Transactions on Consumer Electronics. A zigbee- based home automation system, vol. 55, no. 2, 422- 430 .

[5] Sriskanthan N., Tan F., and Karande A. (2002) Microprocessors and Microsystems. Bluetoothe based home automation system, vol. 26, no. 6, 281-289. Al-Ali A.R. and Al-Rousan M.(2004) IEEE Transactions on Consumer Electronics.Java-based home automation system, vol. 50, no. 2, 498-504.

[6] Ardam H. and Coskun I.(1998) IEEE Transactions on Consumer Electronics. A remote controller for home and office appliances by telephone, vol. 44, no. 4, 1291-1297.

[7] Heierman E.O. III and Cook D.J. (2003) Proc. of Int. Conf. on Data Mining, Improving home automation by discovering regularly occurring device usage patterns,537-540.

[8] Grid-Control web site, www.grid- Control.com/Heimautomatisierung.htm (accessed 24 November 2013).

[9] Fukuoka Smart House web site, www.smartenergy.co.jp/fukuoka/ (accessed 24 November 2013). 